Service social

\title{
L'échelle de développement Harvey, par Maurice Harvey, Brossard, Behaviora, 1984, 64 pages.
}

\section{Francine Ouellet}

Volume 35, numéro 1-2, 1986

Recherche - Action - Évaluation

URI : https://id.erudit.org/iderudit/706308ar

DOI : https://doi.org/10.7202/706308ar

Aller au sommaire du numéro

Éditeur(s)

École de service social de l'Université Laval

ISSN

1708-1734 (numérique)

Découvrir la revue

Citer ce compte rendu

Ouellet, F. (1986). Compte rendu de [L'échelle de développement Harvey, par Maurice Harvey, Brossard, Behaviora, 1984, 64 pages.] Service social, 35(1-2), 272-273. https://doi.org/10.7202/706308ar d'utilisation que vous pouvez consulter en ligne.

https://apropos.erudit.org/fr/usagers/politique-dutilisation/ 
variété des questions soulevées et l'originalité des solutions avancées, nul doute que ces livres constitueront un jalon dans l'évolution du service social nordaméricain. Au-delà des différences, tous(tes) concourent à un objectif commun, les uns avec plus d'optimisme, les autres avec quelque déception, les autres avec scepticisme : il est urgent de travailler à réduire la dépendance du service social vis-à-vis les autres sciences sociales. Les emprunts ne sont pas une catastrophe en tant que telle : la médecine emprunte à la chimie et à la biologie, la sociologie s'inspire de la psychologie, l'anthropologie utilise l'histoire et la science politique, et la psychologie du comportement se combine à la physiologie. Un emprunt devient cependant une dette lorsque l'emprunteur se montre incapable de le faire profiter! C'est le sujet que ces congrès voulaient approfondir une fois de plus, et ils ont réussi à le faire de façon nouvelle.

Département des sciences humaines,

Jean-Pierre DesLAuriers

Université du Québec à Chicoutimi.

L'échelle de développement Harvey, par Maurice HARVEY, Brossard, Behaviora, 1984, 64 pages.

Maurice Harvey, docteur en psychologie, a œuvré principalement dans les hôpitaux et centres d'accueil. Son travail d'intervention, auprès de personnes handicapées mentales, est passé d'une approche clinique, thérapeutique, à une approche éducative. De ce fait, l'élaboration d'outils adaptés autres que les tests psychologiques habituels s'avérait nécessaire.

"L'échelle de développement " a donc été conçu dans le but de répondre aux besoins des intervenants de ce secteur. Elle est avant tout un moyen d'évaluation des progrès du handicapé mental. La première forme de l'échelle a été développée en 1966. Utilisée d'abord localement, elle subissait une première refonte en 1972 et aboutissait sur le marché public en 1974. Depuis ce temps, l'outil ainsi connu a été testé, validé, dans différentes institutions (centres d'accueil et écoles). Maurice Harvey nous présente donc maintenant une échelle améliorée qui tient compte des résultats de ces diverses expérimentations.

Dans l'esprit de l'auteur, il s'agit avant tout d'un manuel pratique plutôt qu'un ouvrage théorique formel. C'est une échelle de mesure des acquis et c'est aussi une mesure du rendement présent du déficient, évalué selon cinq catégories : la motricité, l'autonomie, le graphisme, le langage, les connaissances. Chacune de ces catégories est décortiquée en indicateurs, qui sont directement observables, à l'aide d'un cahier d'items numérotés et relativement simple d'utilisation.

Cet instrument sert principalement à situer l'âge global de développement (A.G.D.), comparativement à l'âge chronologique, et à déterminer un indice évolutif (I.D.). Il s'avère fort intéressant pour un intervenant d'évaluer l'impact 
de son action éducative et ce, à l'aide de l'analyse des acquis. L'utilisation répétée de l'échelle de développement permet donc d'avoir des mesures du progrès du déficient. Cette forme d'utilisation est tout à fait justifiée dans une perspective formative. En effet, le repérage d'une difficulté manifeste est d'autant plus possible et fondée qu'elle s'observe de façon successive, à l'aide du même outil. Dans une intervention de rééducation, cette approche est fort pertinente.

L'auteur nous mentionne que son échelle peut aussi être utilisée pour évaluer les niveaux moyens de groupes. Dans ce contexte, on vise à une meilleure concordance entre les programmes d'intervention dispensés et les capacités réelles des bénéficiaires. C'est donc un moyen qui permet la comparaison, mais attention! L'utilisation normative qui peut en être faite est douteuse, voire même dangereuse. Il suffit de se rappeler la forme de classement qui a prévalu longtemps dans le réseau scolaire primaire, et qui permettait de regrouper en classes homogènes les enfants dits " forts ", " moyens " ou " faibles "! Il faut donc être très prudent quant à l'utilisation d'un tel outil, principalement en ce qui a trait à l'objectif poursuivi.

Sur le plan métrologique, L'échelle de développement s'avère avoir passé tous les examens d'usage. La fidélité de l'instrument a été scrupuleusement contrôlée à l'aide d'un test-retest. Pour éprouver sa validité, on a par la suite soumis l'échelle à une analyse de consistance interne. Son coefficient d'homogénéité calculé, tant intra-catégories qu'inter-catégories, nous semble justifié.

Toujours dans cet esprit de validation, Harvey nous offre une mise en parallèle de son échelle avec quelques autres instruments d'évaluation et de programmation, tels que l'" Adaptive Behavior Scale ", le "Social Maturity Scale " etc. Cette comparaison, fort louable, est intéressante quoique peu poussée.

Ce volume est donc une présentation formelle d'un outil d'évaluation du rendement présent des enfants déficients. C'est un guide pratique à l'usage des intervenants et formateurs. On y trouve, tant les fondements que l'échelle ellemême, soit le cahier d'items par catégories, voire même le feuillet-réponses. II est écrit en langage simple, clair et succinct. Comme tout autre outil de cet acabit, il peut s'avérer fort utile à l'intervenant, en autant qu'il en fait un usage modéré et justifié. Il y a tellement à dire sur l'abus, dans ce domaine, qu'on ne saurait trop inviter le lecteur à la prudence. Bref, un instrument simple, utile, dont les qualités métrologiques ne sont pas à mettre en cause, mais dont l'objectif et la façon de l'utiliser seront constamment à revoir.

Francine OUeLlet

École de service social,

Université Laval. 